\title{
Direct Observation of Tunnelling in KDP Using Neutron Compton Scattering
}

\author{
G.F.Reiter \\ Physics Department and Texas Center for Superconductivity \\ University of Houston, Houston, Texas, USA \\ J. Mayers \\ Rutherford -Appleton Laboratory, Didcot, England \\ P. Platzman \\ Bell Labs, Murray Hill, New Jersey, USA
}

\begin{abstract}
Neutron Compton Scattering measurements presented here of the momentum distribution of hydrogen in $\mathrm{KH}_{2} \mathrm{PO}_{4}$ (KDP) just above and well below the ferroelectric transition temperature show clearly that the proton is coherent over both sites in the in the high temperature phase, a result that invalidates the commonly accepted order-disorder picture of the transition. The BornOppenheimer potential for the hydrogen, extracted directly from data for the first time, is consistent with neutron diffraction data, and the vibrational spectrum is in substantial agreement with infrared absorption measurements. The measurements are sensitive enough to detect the effect of surrounding ligands on the hydrogen bond, and can be used to study the systematic effect of the variation of these ligands.
\end{abstract}

s PACS numbers, 61.12-q, 64.60-i,77.80-c

It is known that in KDP [1]

each proton is equally likely to be in either of two positions in the hydrogen bond above the ferroelectric phase transition at $\mathrm{Tc}=124 \mathrm{~K}$, where the structure is tetragonal, and is nearly entirely in one site below Tc, where the structure is orthorhombic. What has not been known, and which has been a subject of controversy since Blinc [2] proposed the idea in 1973, is whether the protons are tunnelling between the two equivalent sites or are localized in one or the other of the two sites and simply disordered above the transition. While the tunnelling model was used to describe the data for some time after it was proposed, Raman scattering data [3] that seemed to show that the symmetry of the surroundings of the proton did not change above and below the transition, together with subsequent neutron scattering results [ [A], led to the consensus that the proton was self trapped in one or the other of its equivalent positions, and jumped from position to position through phonon assisted tunnelling. If such a picture were correct, the proton would be in one site or the other in the high temperature phase for times much longer than the time scale of our experiment $\left(10^{-15} \mathrm{sec}\right)$, not simultaneously in both. The transition would be an order-disorder type in which the populations of the two sites became unequal below the ordering temperature. As pointed out by Reiter and Silver [5], neutron Compton scattering provides a means to distinguish between the tunnelling and the order disorder model. If the latter were correct, NCS should see only a small change in the momentum distribution. This would be due to whatever changes occurred in going through the transition to the potential of individual wells in which the proton was trapped. By contrast, if the particle were tunnelling and then became trapped, we should see a narrowing of the momentum distribution in the tunnelling phase, together with an oscillation due to the coherent interference of the proton in the two sites. We show here that the distributions at temperatures above and below the ferroelectric transition are, in fact, dramatically different, and consistent with the proton coherently occupying the two sites. Since the bond is symmetric above the transition, one can construct the effective Born-Oppenheimer potential directly (without any model) from the measurement of the momentum distribution [5]. This is as far as we know, the first such measurement of a Born-Oppenheimer potential in any system. It yields a double well potential with parameters consistent with neutron crystallography and incoherent neutron scattering at lower scattering energies, and yields vibration frequencies consistent with infrared measurements. The experiments are done on the electron volt spectrometer, EVS, at ISIS, the pulsed neutron source at the Rutherford Laboratory. This sort of source is needed to provide high energy neutrons (5-10 ev) for which the energy transfer is sufficiently large compared to the characteristic energies of the system that the scattering is given accurately by the impulse [6] approximation limit. The scattering at these energies is entirely incoherent, each particle scattering independently. $S_{M}(\vec{q}, \omega)$, the scattering function for a particle of mass $\mathrm{M}$, is related to the momentum distribution of the particle $\mathrm{n}(\vec{p})$ in this limit by the relation

$$
S_{M}(\vec{q}, \omega)=\int n(\vec{p}) \delta\left(\omega-\frac{\hbar q^{2}}{2 M}-\frac{\vec{p} \cdot \vec{q}}{M}\right) d \vec{p}
$$


where $\hbar \omega$ is the energy transfer, $M$ is the mass of the proton, and $q=|\vec{q}|$ is the magnitude of the wave-vector transfer.

The small mass of the proton leads to a broad distribution in energy of the scattered neutrons, centered at $\frac{\hbar^{2} q^{2}}{2 M}$, that is well separated from the scattering from the heavier ions such as oxygen, which appear as nearly elastic contributions. This, together with its large incoherent cross-section, make it an ideal candidate for these measurements. $n(\vec{p})$, the probability of observing the proton with momentum $\vec{p}$, for simple one particle systems in their ground state, is the square of the absolute value of the fourier transform of the spatial wave-function. The experimental data is fit in a model free way using a series expansion that allows one to reconstruct $\mathrm{n}(\mathrm{p})$ directly from the fitted coefficients. We represent $S_{M}(\vec{q}, \omega)$ as $\frac{M}{q} J(\hat{q}, y)$ where $\mathrm{y}=\frac{M}{q}\left(\omega-\frac{q^{2}}{2 M}\right)$, and expand $J(\hat{q}, y)$ as

$$
J(\hat{q}, y)=\frac{e^{-y^{2}}}{\pi^{\frac{1}{2}}} \sum_{n, l, m} a_{n, l, m} H_{2 n+l}(y) Y_{l m}(\hat{q})
$$

where the $H_{n}(y)$ are Hermite polynomials and the $Y_{l m}$ are spherical harmonics. This series is truncated at some $\operatorname{order}(2 \mathrm{n}+\mathrm{l}=10)$ in this case), convolved with the instrumental resolution function and then least squares fit to the data. The coefficients $a_{n, l, m}$ then determine the measured $n(\vec{p})$ directly as a series in Laguerre polynomials and spherical harmonics [5],7] The procedure is a smoothing operation, which works with noisy data, and which also allows for the inclusion of small corrections to the impulse approximation [7, \&]. The errors in the measured $n(\vec{p})$ are determined by the uncertainty in the the measured coefficients, through their correlation matrix, which is calculated by the fitting program. For an expanded discussion of the procedure and errors see Ref 7 . In fitting the data, we take the $\mathrm{z}$ axis to be along the bond axis, the $\mathrm{x}$ axis to be the $\mathrm{c}$ axis of the crystal, perpendicular to both bonds, and fit the data with the sum of two terms, one rotated by 90 degrees about the $\mathrm{x}$ axis from the other. The measurement provides the complete $3-\mathrm{D}$ momentum distribution. We show in Fig. 1 a cut along the $p_{x}-p_{z}$ plane for the momentum distribution of a single bond at two temperatures, one far below and one just above the transition. The measurements were taken with exactly the same experimental configuration, and analyzed with exactly the same terms kept in the series expansion. If the order-disorder picture of the transition had been correct, we would expect to see small changes in the momentum distribution on going through the transition, as the proton would remain localized in one or the other equivalent sites, and the confining potential at that site would be only slightly modified. In fact we see dramatic changes in the width and the shape of the momentum distribution. While there is little change in the direction normal to the bond, the distribution along the bond changes qualitatively. 
a) KDP Momentum Distribution $\mathrm{T}=90 \mathrm{~K}$

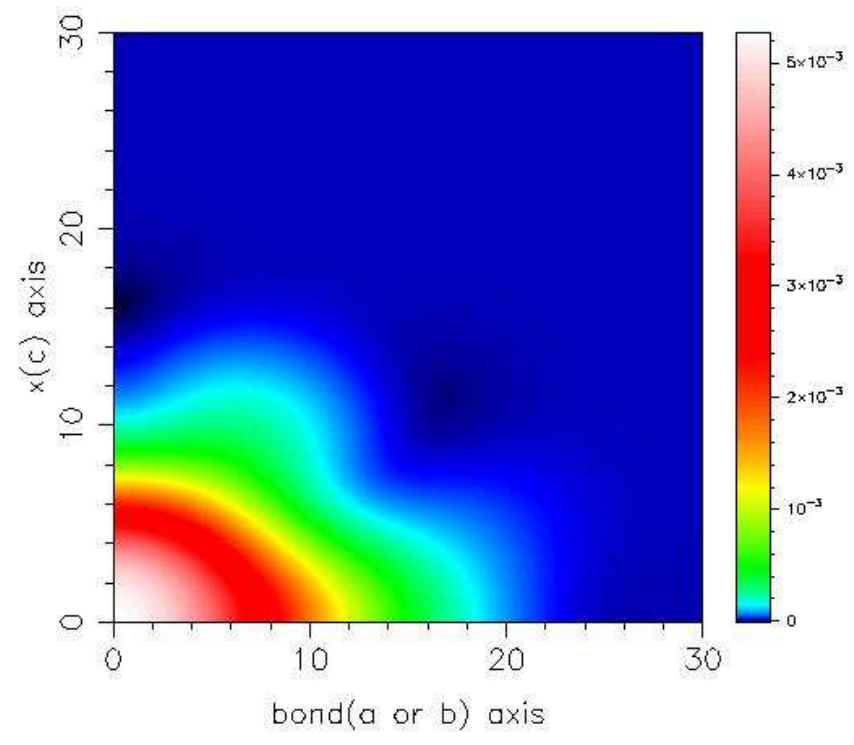

b) KDP Momentum Distribution $\mathrm{T}=130 \mathrm{~K}$

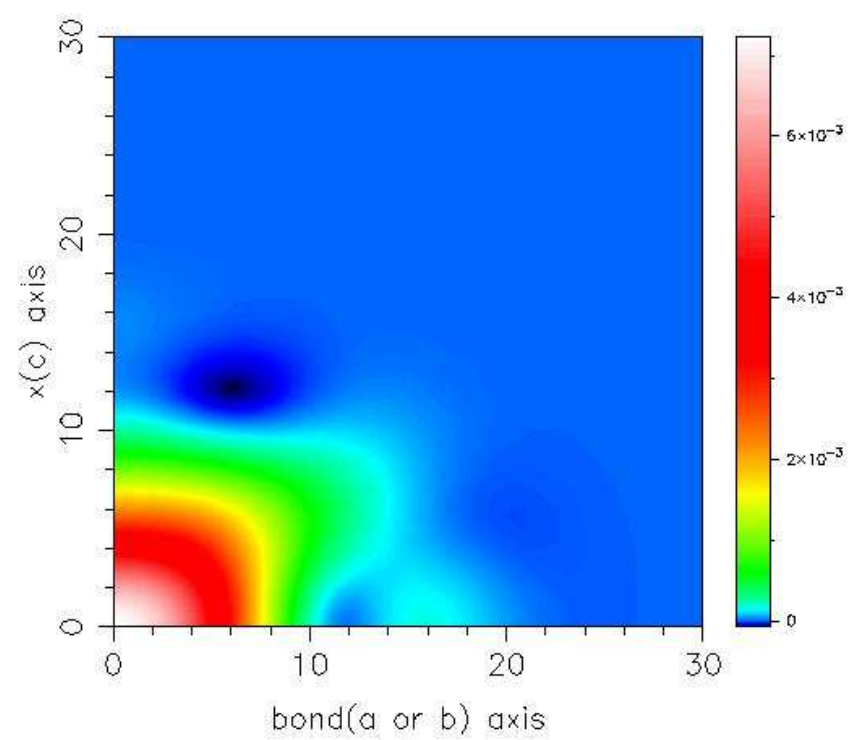

FIG. 1. The momentum distribution in the $p_{x}-p_{z}$ plane for a single hydrogen bond below $(\mathrm{T}=90 \mathrm{~K})$ and just above(T=130K) the ferroelectric transition at $\mathrm{T}=124 \mathrm{~K}$. The bulge in (b) along the direction roughly 30deg from the bond axis, that is the projection of the displacement from the center of the bond to the phosphorus ion, is interpreted as being due to the repulsion of the proton by the phosphorus.

The momentum distribution along the bond axis for both temperatures is shown in Fig. 2. The dotted lines give the uncertainty in the distribution function as a result of the uncertainty in the measured coefficients. 
KDP Momentum Distribution Along Bond

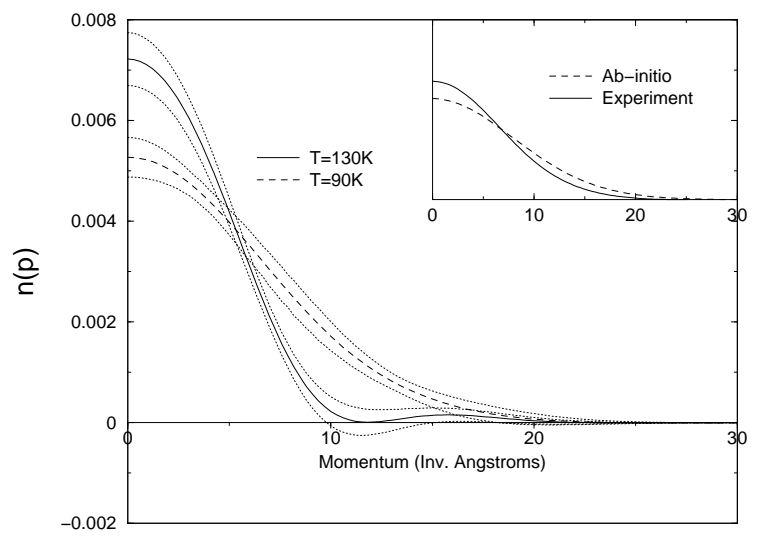

FIG. 2. The momentum distribution along the bond axis for temperatures just above and well below the structural phase transition at $124 \mathrm{~K}$. The dotted curves surrounding the heavy lines are one standard deviation error markers. The inset is a comparison of the measured momentum distribution at $\mathrm{T}=90 \mathrm{~K}$ with a recent calculation by Koval, Kohanoff and Migoni[11] in which their ab-initio one particle potential along the bond was used to calculate the momentum distribution from the one dimensional Schrodinger equation,assuming the three dimensional potential was separable.

We see that overall, there is considerable narrowing of the distribution in the high temperature phase, indicating an increase in the length along the bond over which the spatial wave function is coherent. The prominent feature of the high temperature distribution, the zero and the subsequent oscillation, is precisely what one would expect for a spatial wave-function that was coherent over both sites, with the position of the zero being determined by the separation of the sites.

The momentum distribution contains many body effects due to the motion of the surrounding ions. These effects are quite small for the heavy ions [12], but could be significant for the interaction of the protons with themselves. If we treating them in a mean field approximation, we can calculate an effective one body Born-Oppenheimer potential from the momentum distribution in the high temperature phase, assuming the proton is in its ground state [5]. We will assume the potential is separable to simplify this calculation, so that we only need the data along the $p_{z}$ axis.

The error bars on the measurements are such that there is considerable uncertainty in the tail of the distribution, and one could argue that there is no oscillation. If, however, we take the zero of the most probable distribution to be real, and choose the sign of the wave-function as negative for momenta greater than the momentum at the zero, then we find the effective Born-Oppenheimer potential and the spatial wave function shown in Fig. 3. If we do not change the sign, we get a completely un-physical potential. It is clear that the most likely momentum distribution measured supports a tunnelling model. The tunnel splitting is $94 \mathrm{mev}(\sim 1000 \mathrm{~K})$, so that our assumption that we are seeing only the ground state is consistent. Even if one doesn't take seriously the oscillation in the tail of the momentum distribution, and assumes that the actual distribution goes smoothly to zero, we would find a much broader potential above $\mathrm{T}_{c}$ than below, and the order-disorder model would be equally untenable. That potential would, however, be inconsistent with the neutron crystallography [1], which clearly shows a double peak structure for the spatial wave-function, as in Fig. 3. 


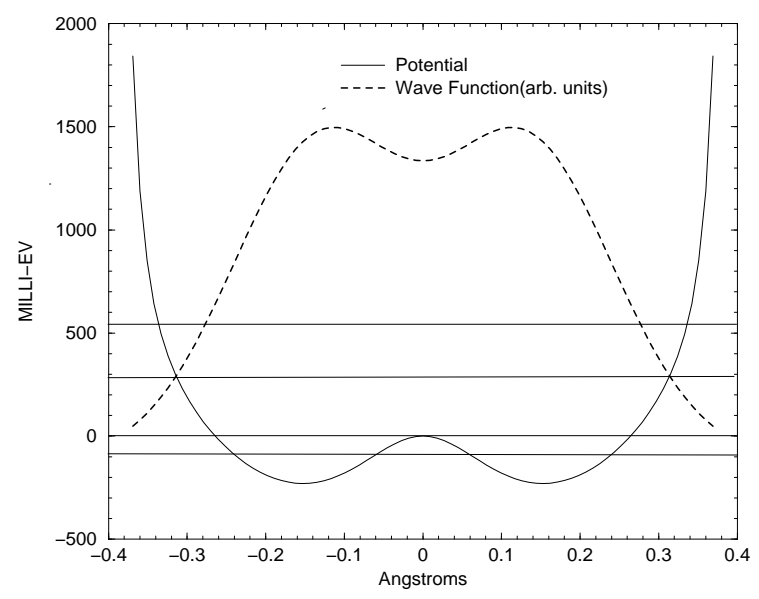

FIG. 3. The effective Born-Oppenheimer potential that corresponds to the measured momentum distribution along the bond at $\mathrm{T}=130 \mathrm{~K}$, (Fig. 2), together with the spatial wave function for that potential. The horizontal lines give the first four energy levels for the measured potential.

Although the potential in Fig. 3 is quite different from that inferred from infrared absorption data by Lawrence and Robertson [13 who assumed that it could be described by back-to-back Morse potentials, the transition energies they based the fit on are substantially the same as those in the figure. LR identify the $0 \rightarrow 3$ transition as having an energy of $4600 \mathrm{~cm}^{-1}$, whereas we obtain $5088 \mathrm{~cm}^{-1}$. For the $1 \rightarrow 2$ transition, LR obtain $2260 \mathrm{~cm}^{-1}$ while we obtain 2039 $\mathrm{cm}^{-1}$. Neutron crystallography studies have fit the shape of the spatial wave-function with the sum of two displaced Gaussians, which would give a rather different potential than that of Fig. 3, and obtained a separation of the minima of the potential, of .34 Angstroms. We find .31 Angstroms for this separation. The shape of the spatial wave function in Fig. 3 is qualitatively similar to that described in Ref. 1 . We conclude that the potential of Fig. 3 is at least qualitatively correct, and that the proton is indeed tunnelling between equivalent sites in the high temperature phase.

Below the transition, since the potential is not symmetric, it is not possible to invert the data to obtain it, as phase information is lost in the momentum distribution. If one has a prediction for the potential, one can, however, calculate the momentum distribution. In the inset of Fig. 2, we show the momentum distribution obtained from a recent 11] ab-initio calculation of the Born-Oppenheimer potential for KDP in which the electronic many-body problem was treated by density functional theory. The one particle approximation is evidently reasonably good here. The same calculations shows that there are significant interactions amongst the hydrogens above $T_{c}$, so that an unambiguous one particle potential cannot be obtained theoretically, as the potential for the motion of a single particle depends sensitively on the positions assumed for the surrounding hydrogen. The present measurements provide a means of testing theoretical inclusions of these many body effects on the effective one particle potential, which should reproduce the observed momentum distribution.

If a hydrogen bond were isolated, the momentum distribution would have to be symmetric about the bond axis. We find that distribution in the $p_{x}-p_{y}$ plane, (not shown here) is significantly broader at 45 degrees to either axis than it is along the axes. This direction is approximately the projection in the $\mathrm{x}-\mathrm{y}$ plane of the vector from the center of the bond to the phosphorus ion [14]. If we return to Fig. 1, we see that there is also a broadening of the distribution in the high temperature phase at approximately 30 degrees to the $\mathrm{z}$ axis, which is the approximate direction of the projection of the vector from the center of the bond to the phosphorus ion on the x-z plane. This broadening becomes a narrowing at low temperatures. We conclude that this broadening in momentum space is a result of the repulsion of the proton by the phosphorus ion, and that the transition displaces the particles in a way that reduces that repulsion. This is in broad agreement with both the calculation cited above and another recent ab-initio calculation [15] that showed that the motion of the phosphorus had large effects on the hydrogen potential surface. We conclude that the experiments are easily sensitive enough to see the effect of the surrounding ions on the hydrogen bond, and include many body effects that are difficult to calculate with existing methods. This sensitivity opens up the possibility of studying the effect of the systematic variation of the surrounding ligands on the dynamics of hydrogen bonds. The knowledge gained could then be used to identify the best models and ab-initio approximation schemes, and to infer the behavior of the bonds in environments where the measurements can't be done because there are two many inequivalent hydrogens, such as in DNA or proteins. 


\section{ACKNOWLEDGMENTS}

We would like to thank Devinder Sivia for assistance in analyzing the data, Richard Nelmes, Jorge Kohanoff , Nicholas Kioussis and Naresh Dalal for useful discussions, and Jorge Kohanoff for permission to use the results of Ref. 11 .

[1] R. Nelmes, Ferroelectrics 71, 87, (1987)

[2] R. Blinc, J. Phys. Chem. Solids 13,204,(1960)

[3] Y. Tominaga, H. Urabe and M. Tokunaga, Solid. St. Comm. 48, 265, 1983

[4] S. Ikeda and Y. Yamada, Physica B, 213, 652, (1995)

[5] G. Reiter and R. Silver, Phys. Rev. Letts. 54, 1047 (1985)

[6] P. M. Platzmann, in Momentum Distributions, ed R. N. Silver and P. E. Sokol (Plenum Press,New York, 1989, p249)

[7] G. Reiter, J. Mayers, and J. Noreland, ( to be published in Phys. Rev. B)

[8] V. F. Sears, Phys. Rev. 185,200, (1969);Phys. Rev. A7, 340, (1973)

[9] G. Felcher and I. Pelah, J. Chem. Phys. 52, 905, 1970

[10] E. Wiener, S. Levin and I. Pelah, J. Chem. Phys. 52, 2881, (1970)

[11] S. Koval, J. Kohanoff and R. Migoni, (to be published in Ferroelectrics )

[12] M. Warner, S. W. Lovesey, , and J. Smith, Z. Phys. B 39, 2022 (1989)

[13] M. C. Lawrence and G. N. Robertson, Ferroelectrics, 34, 179, (1981)

[14] Nelmes,R.,J., Tun, Z.,and Kuhs,W.,F., Ferroelectrics, 71, 125, (1987)

[15] Zhang,Q.,Chen,F.,and Kioussis, N., Phys. Rev. B 65, 24108,(2002) 